\title{
Evaluación de la Competencia Terapéutica: Consistencia Interna y Acuerdo Inter-Jueces de la Escala de Terapia Cognitiva (CTS $)^{7}$
}

\author{
Leticia Maurino \\ Licenciada en Psicología \\ Universidad Nacional de Córdoba \\ Argentina \\ Correo electrónico: leticiamaurino.Im@gmail.com
}

Ana Laura Miranda

Licenciada en Psicología Universidad Nacional de Córdoba

Argentina

Correo electrónico: mirandanalaura@gmail.com

\section{Luciana Moretti}

Doctora en Psicología Universidad Nacional de Córdoba Argentina Correo electrónico: Ismoretti@gmail.com

\author{
Pablo Ezequiel Flores-Kanter \\ Licenciado en Psicología \\ Universidad Nacional de Córdoba \\ Argentina \\ Correo electrónico: ezequielfk@gmail.com
}

Leandro Casari

Doctor en Psicología

Consejo Nacional de Investigaciones Científicas y Técnicas (CONICET)

Argentina

Correo electrónico: Imcasari@ mendoza-conicet.gov.ar

\author{
Leonardo Adrián, Medrano \\ Doctor en Psicología \\ Universidad Nacional de Córdoba \\ Argentina \\ Correo electrónico: leomedpsico@gmail.com
}

Recibido: 01/06/2018

Evaluado: 02/10/2018

Aceptado: 27/11/2018

\section{Resumen}

Contar con instrumentos fiables para medir la competencia de los terapeutas en el contexto de la terapia cognitivo-conductual es una herramienta de gran valor a nivel clínico y empírico. La Escala de Terapia Cognitiva (CTS) es una de las escalas más ampliamente utilizadas para medir la competencia terapéutica. A nivel internacional son escasos los antecedentes que se han propuesto indagar su validez y confiabilidad, y sigue siendo éste un tema controvertido, particularmente la confiabilidad interjueces. Sumado a lo anterior, no se encuentran investigaciones de este tipo en Argentina, motivo por el cual el presente trabajo tuvo como principal objetivo indagar la confiabilidad de la escala CTS. Para esto se conformó una muestra de 10 jueces expertos, los cuales, mediante la CTS, evaluaron el desempeño que presentaban diferentes terapeutas $(n=4)$ durante el desarrollo de una sesión. Los resultados permitieron concluir que se trata de un instrumento confiable. En efecto, se apreció una consistencia interna óptima, tanto en la correlación ítem-total como en el coeficiente alfa de Cronbach. Asimismo, en general se observó una buena consistencia en las puntuaciones dadas por los diferentes profesionales. Los datos presentados en esta investigación avalan la utilización de la escala en el contexto argentino y permiten señalar que la CTS es una escala confiable.

\section{Palabras clave}

Escala de Terapia Cognitiva, Competencia Terapéutica, Consistencia Interna, Confiabilidad Interjueces. 


\section{Evaluation of the Therapeutic Competence: Internal Consistency and Inter-Judges Agreement of the Cognitive Therapy Scale (CTS)}

Abstract

Having reliable instruments to measure the competence of therapists in the context of cognitive-behavioral therapy is a tool of great clinical and empirical value. The Cognitive Therapy Scale (CTS) is one of the scales most widely used to measure therapeutic competence. In the international arena, there are few antecedents that have been proposed to investigate their validity and reliability, and this is still a controversial issue, particularly inter-judge reliability. In addition to the above, there is no research of this type in Argentina, which is why the main objective of this work was to investigate the reliability of the CTS scale. For this, a sample of 10 expert judges was formed, who, through the CTS, evaluated the performance presented by different therapists $(n=4)$ during the development of one session. The results allowed to conclude that it is a reliable instrument. In effect, an optimal internal consistency was observed, both in the item-total correlation and in the Cronbach alpha coefficient. Likewise, in general, a good consistency was observed in the scores given by the different professionals. The data presented in this research support the use of the scale in the context of Argentina and allow us to point out that the CTS is a reliable scale.

Keywords Cognitive Therapy Scale (CTS), therapeutic competence, internal consistency, interjudge reliability.

\section{Avaliação da Competência Terapêutica: Consistência Interna e acordo interjueces da Escala de Terapia Cognitiva (CTS)}

Resumo

Ter instrumentos confiáveis paramedir a competência dosterapeutasno contexto daterapia cognitivo-comportamental é uma ferramenta de grande valor em um nível clínico e empírico. A Cognitive Therapy Scale (CTS) é uma das escalas mais utilizadas para medir a competência terapêutica. No nível internacional, existem poucos antecedentes que foram propostos para investigar sua validade e confiabilidade, e essa ainda é uma questão controversa, particularmente a confiabilidade entre os julgamentos. Além do exposto acima, não existe pesquisa desse tipo na Argentina, motivo pelo qual o presente trabalho teve como principal objetivo investigar a confiabilidade da escala CTS. Para isso, formou-se uma amostra de 10 juízes especialistas que, por meio do CTS, avaliaram o desempenho apresentado por diferentes terapeutas $(n=4)$ durante 0 desenvolvimento de uma sessão. 0 s resultados permitiram concluir que é um instrumento confiável. Com efeito, foi observada uma consistência interna ótima, tanto na correlação item-total quanto no coeficiente alfa de Cronbach. Da mesma forma, em geral, observou-se boa consistência nas pontuações dadas pelos diferentes profissionais. Os dados apresentados nesta pesquisa apoiam o uso da escala no contexto argentino e nos permitem apontar que a CTS é uma escala confiável.

Palavras chave

Escala de Terapia Cognitiva, competência terapêutica, consistência interna, confiabilidade interjueces. 


\section{ntroducción}

El movimiento de prácticas basadas en la evidencia refiere a la integración entre la mejor evidencia disponible con la experiencia clínica tomando en consideración las circunstancias y características únicas de cada paciente (American Psychological Association [APA], 2005). Inicialmente este modelo estuvo centrado en demostrar la efectividad de la psicoterapia sobre la ausencia de ésta. En un segundo momento, se estudió el efecto de las variables específicas de cada método terapéutico, es decir, se buscó determinar la efectividad diferencial de las técnicas utilizadas, intentando distinguir los enfoques que reunían mayor evidencia empírica. Actualmente se desarrolla una nueva etapa, en la cual la investigación está orientada hacia las variables inespecíficas de la psicoterapia. Esto es, variables comunes a los diferentes enfoques terapéuticos que no tienen que ver con el método o técnicas aplicadas, sino con aspectos del paciente, del terapeuta y de la relación terapéutica (Webb, DeRubeis \& Barber, 2010; Winkler, Cáceres, Fernández \& Sanhueza, 1989).

El interés por el estudio de los factores inespecíficos se debe al impacto que poseen estas variables sobre el cambio terapéutico, señalada como responsables de un mayor porcentaje del cambio terapéutico que las variables específicas (Romero, 2008). Específicamente, las competencias y características personales del psicoterapeuta tienen un efecto ocho veces mayor en el resultado de la psicoterapia que la orientación teórica o las técnicas utilizadas para el tratamiento (Araya \& Herrera, 2007). Sobre el terapeuta, un estudio concluyó que niveles más altos de adhesión al modelo terapéutico y mejores medidas de competencia terapéutica, fueron asociados con mejores resultados terapéuticos (Webb et al., 2010). Estudiar cuáles son las características del terapeuta que están relacionadas con la eficacia del proceso terapéutico, cobra relevancia no solamente por su influencia en el éxito o fracaso de la terapia, sino porque su conocimiento tiene una repercusión directa en la formación de nuevos profesionales (Rodríguez \& Salinas, 2011).

El estudio de los aspectos del terapeuta ha sido demasiado amplio y no se ha centrado en un mismo constructo (Casari, Ison, Albanesi \& Maristany, 2017). Es difícil encontrar modelos útiles acerca del desarrollo de habilidades terapéuticas para guiar a los investigadores o instructores (Bennett-Levy, 2006). Distintos autores han utilizado diferentes marcos para describir los elementos clave de habilidades terapéuticas, y no existe un consenso sobre las habilidades y características que determinan el nivel de competencia del terapeuta (Rakovshik \& McManus, 2010; Rodríguez \& Salinas, 2011).

Entre las variables que se han señalado como influyentes en el cambio desde la perspectiva del terapeuta se encuentran: habilidades comunicacionales, interés genuino por las personas y su bienestar, autoconocimiento, compromiso ético, calidez, empatía, respeto por el paciente, optimismo, sentido del humor, capacidad de auto cuestionarse, flexibilidad, capacidad para establecer una alianza de trabajo, dominio de técnicas 
específicas, igualdad étnica, competencias multiculturales y entrenamiento (Heinonnen, 2014; Holdsworth, Bowen, Brown \& Howat, 2014; Nissen-Lie, Havik, Høglend, Monsen, \& Rønnestad, 2013). Así, dentro de las variables sobre las que no hay consenso, pueden mencionarse respecto al terapeuta: su edad, origen étnico, género, nivel de experiencia, entrenamiento, tipo de formación y orientación teórica (Nissen-Lie et al., 2013).

Existen otros factores que son más controversiales, mostrando contradicciones entre diversos modelos terapéuticos respecto a cuáles son las características que se desean y debieran entrenarse en un terapeuta. En este sentido, lo que podría considerarse competente para un paradigma psicoterapéutico, puede ser percibido como incompetente e incluso poco ético por otro (Rakovshik \& McManus, 2010).

La falta de acuerdo teórico y conceptual sobre las variables del terapeuta torna compleja la investigación en el tema. Sumado a ello, se observan solapamientos conceptuales que hacen más compleja la comprensión de estos factores. Es habitual que conceptos diferentes como habilidades, competencias y estilos terapéuticos, se utilicen de manera intercambiable o como sinónimos. Para Rodríguez y Salinas (2011) las habilidades terapéuticas refieren a aptitudes y actitudes que debe tener en cuenta un terapeuta para dedicarse a la práctica clínica, que están centradas en su persona y son independientes de su postura teórica. Por otra parte, el estilo personal del terapeuta se define a partir de la interacción entre las funciones y los rasgos o actitudes que un terapeuta evidencia en su ejercicio profesional, y se compone de cinco funciones: instruccional, atencional, expresiva, operativa y de involucración (Fernández-Álvarez, García, Lo Bianco \& Corbella, 2003). Finalmente, las competencias terapéuticas refieren al conjunto de talentos y destrezas teóricas y/o aplicadas sobresalientes en psicoterapia; involucran al sujeto en su totalidad, en los niveles de actividad conductual, emocional y cognitivo (Yáñez-Galecio, 2005). Otros autores (DeRubeis, Hollon, Evans, \& Bemis, 1982; Hill, O'Grady, \& Elkin, 1992) han definido la competencia terapéutica como la habilidad del terapeuta para conceptualizar los problemas del paciente desde un marco teórico específico, aplicando técnicas o métodos reconocidos y congruentes con los objetivos del tratamiento.

La falta de claridad conceptual no permite tener la seguridad de que distintos estudios midan la misma variable. Existen limitaciones en la evaluación de la competencia terapéutica, por lo que resulta necesario realizar refinamientos en cómo es medido y conceptualizado dicho constructo (Strunk, Brottman, De Rubeis, \& Hollon, 2010). En coincidencia con estas ideas, Muse \& McManus (2013) refieren que el conocimiento es el dominio más fácil de operacionalizar y evaluar, mientras que la evaluación de los aspectos más complejos, tales como las habilidades y competencias terapéuticas, han demostrado un mayor desafío.

Por otra parte, algunos autores abordan las competencias terapéuticas como un factor independiente de la postura teórica (Rodríguez \& Salinas, 2011; Yáñez-Galecio, 2005). Otros investigadores consideran que las mismas deben ser congruentes con la orientación teórica, los métodos usados y los objetivos de 
tratamiento (DeRubeis et al., 1982; Hill et al., 1992). A pesar de estas diferencias, es innegable que las habilidades que constituyen la competencia terapéutica varían según el dominio terapéutico.

En un intento de superar la dualidad entre quienes sostienen que las competencias son factores comunes a todos los modelos terapéuticos y quienes distinguen habilidades particulares requeridas para cada corriente terapéutica, resulta útil diferenciar los conceptos de adhesión y competencia. La adhesión refiere el grado en que el terapeuta se adhiere al modelo y principios que guían el tratamiento. Es decir, si utiliza las técnicas y procedimientos propuestos por el modelo terapéutico, o, si por el contrario, utiliza un abordaje ecléctico o personal que no refleja los principios o procedimientos que sugiere el modelo terapéutico de base. Por otra parte, la competencia refiere a la habilidad con la cual las intervenciones son puestas en práctica (Muse \& McManus, 2013). La aplicación correcta de un tratamiento requiere de la adhesión al modelo de tratamiento y el manejo competente de las técnicas especificadas. Así, la competencia no es suficiente sin la adhesión (p.ej., la entrega experta de técnicas de un modelo de tratamiento diferente) de la misma manera que la adhesión no es suficiente sin la competencia (p.ej., la entrega rígida de un manual de tratamiento sin ninguna habilidad terapéutica); la adhesión no necesariamente implica la entrega competente del procedimiento. Los autores refieren que hay incertidumbre en cuanto a si la adhesión y la competencia deberían ser medidas por separado o simultáneamente y esto puede depender del contexto de la evaluación. Sin embargo, en el contexto de formación de terapeutas y de la práctica diaria, se recomienda considerar ambos componentes en combinación (Muse \& McManus, 2013).

Teniendo en cuenta que la competencia terapéutica no es por sí sola un indicador de éxito terapéutico, y que es pertinente evaluarla en combinación con la adherencia a un modelo terapéutico, es necesario definir la competencia desde la terapia cognitivo-conductual. Desde esta perspectiva terapéutica, estudiar cuáles son las competencias que predicen el éxito terapéutico cobra vital importancia. Dado el interés que desde este modelo se ha fundado por adherirse a las prácticas basadas en la evidencia, surge la preocupación por conocer cuáles son las habilidades que deberían incluirse en la formación de nuevos terapeutas para maximizar el éxito del proceso terapéutico.

Con el fin de determinar cuáles son las competencias deseables en un terapeuta cognitivo-conductual, Rodríguez \& Salinas (2011) toman aportes de Beck, Rush, Shaw \& Emery (1976) para establecer que son necesarias, aunque no suficientes, tres competencias: aceptación, empatía y autenticidad. Por su parte, Mairal (2009) clasifica las habilidades terapéuticas en: destrezas básicas referidas a la relación terapéutica, habilidades de escucha, habilidades de acción verbal, habilidades del conocimiento sobre el modelo específico desde el que se trabaja, competencias sobre las técnicas concretas, y otras habilidades más generales y difíciles de clasificar tales como fluidez, flexibilidad, entre otras. Por último, Roth \& Pilling (2007) identifican las competencias principales requeridas para la aplicación eficaz de 
terapia cognitivo-conductual y encontraron más de cincuenta competencias específicas agrupadas dentro de cinco dominios: competencias terapéuticas genéricas (p.ej., conocimiento de problemas de salud mental, capacidad de recibir a un paciente); competencias básicas (p.ej., conocimiento de principios teórico-técnicos, estructura de la intervención); técnicas específicas (p.e., descubrimiento guiado, empleo de registros de pensamiento); competencias específicas de cada problema (p.e., competencias que son descritas en manuales de tratamiento para desórdenes específicos, procedimientos específicos que han demostrado científicamente ser eficaces para problema particulares); y metacompetencias (realizar una tarea experta requiere que la persona aplique el modelo apropiadamente, son los procedimientos utilizados por los terapeutas para guiar la práctica, a través de todos los niveles del modelo).

A pesar del desacuerdo conceptual en torno a la competencia terapéutica, los estudios empíricos sobre la formación en competencias arrojan resultados esperanzadores. Shaw \& Wilson-Smith (1988) evaluaron la habilidad terapéutica utilizando la Escala de Terapia Cognitiva (CTS) (Young \& Beck, 1980) y hallaron un aumento muy significativo en la competencia terapéutica global luego del entrenamiento. En otro estudio empírico, James, Blackburn, Milne \& Reichfelt (2001) utilizaron la escala CTS-R para evaluar la competencia de veinte alumnos de postgrado, en tres ocasiones durante el curso de formación de terapia cognitivo conductual (TCC) y encontraron que la competencia se incrementó significativamente durante el período de formación, lo que sugiere que el curso fue eficaz en el desarrollo de competencias. Por su parte, Myles \& Milne (2004) evaluaron un programa de formación en TCC de 48 horas usando múltiples medidas y un diseño de grupo control con 90 participantes; concluyendo que el entrenamiento fue efectivo en términos de los conocimientos y habilidades que adquirieron los participantes, y de la generalización de este aprendizaje al ambiente de trabajo. En la misma línea de trabajo, Barnfield, Mathieson \& Beaumont (2007) estudiaron un programa de formación en TCC y hallaron que el conocimiento de la TCC de los alumnos, la capacidad para conceptualizar y las habilidades terapéuticas mejoraron significativamente como resultado del curso.

No obstante, otros estudios no encontraron resultados alentadores, ya que la competencia del terapeuta no tuvo el grado de relación esperado con los resultados del paciente (Shaw et al., 1999). Las posibles razones de tales hallazgos serían la restricción en el rango de los puntajes de la CTS en las pruebas clínicas y limitaciones de la escala en sí misma (Whisman, 1993). La escala CTS de 11 ítems no sería lo suficientemente completa ni específica para evaluar el constructo de competencia terapéutica cognitiva (Shaw et al., 1999). Las limitaciones que se señalaron incluían el hecho de que algunos ítems son acumulaciones de diferentes constructos que necesitarían ser separados y otros que requerirían un mayor nivel de inferencia.

Como se puede observar en los resultados de investigaciones que anteceden el presente trabajo, una porción menor de los estudios explorados son contradictorios. Esta contradicción 
puede estar relacionada con las limitaciones de los diseños de los estudios y del instrumento (CTS) (Fairburn \& Cooper, 2011); así como también debido a que la CTS original ha demostrado un fuerte acuerdo entre los calificadores para la competencia general, pero sólo moderado para elementos específicos de la escala (Rakovshik \& McManus, 2010). No obstante, como limitaciones, la gran mayoría de los estudios revisados sugieren que las competencias terapéuticas son susceptibles de entrenamiento y aprendizaje. Este hecho justifica la necesidad de continuar investigando cuáles son las competencias del terapeuta que aumentan la eficacia del proceso terapéutico para incluir su entrenamiento en programas de formación para nuevos terapeutas. Para ello, es imprescindible el refinamiento de los instrumentos utilizados para evaluar la competencia. A pesar de las limitaciones, la CTS y las escalas similares siguen siendo el método más ampliamente utilizado para evaluar la competencia del terapeuta en TCC. Su uso generalizado facilita la comparación de la competencia terapéutica entre los estudios.

La CTS es una de las escalas más ampliamente utilizadas con el objeto de medir la competencia terapéutica (Barber, Liese, \& Abrams, 2003; Gordon, 2006; Muse \& McManus, 2013). Sin embargo, existe poca evidencia a nivel internacional respecto a la consistencia interna y validez de la CTS, ya que los estudios son escasos (Barber et al., 2003; Vallis, Shaw \& Dobson, 1986). De dichas investigaciones puede concluirse que la CTS demuestra una consistencia interna óptima (valores $\alpha$ entre .84 y .95; y correlaciones ítem-total entre .48 y .94), así como una estructura bifactorial
(Muse \& McManus, 2013; Vallis et al., 1986). Sin embargo, los resultados respectos a la confiabilidad del instrumento no demuestran datos consistentes, particularmente respecto a la confiabilidad interjueces. En efecto, mientras algunos estudios dan cuenta de valores adecuados de confiabilidad interjueces (Barber et al., 2003; Gordon, 2006), otros estudios han evidenciado coeficientes entre bajos y moderados (Vallis et al., 1986). Reichelt, James, \& Blackburn (2003) sintetizan estos antecedentes e indican que las correlaciones interclase obtenidas se encuentran entre los valores .54 y .96, aunque si uno considera este indicador de confiabilidad en cada ítem los valores van del .27 al .59. Sin embargo, un estudio reciente de Muse y McManus (2013) señaló que para el puntaje CTS total se han observado confiabilidades interjueces de magnitudes variables, desde acuerdo pobre (ICC $=.01-.08)$ y moderado (ICC $=.47-.59$ ), a acuerdo bueno (ICC = .69-.77) y muy bueno (ICC = .83-.94). Asimismo, en el caso de los ítems del CTS la confiabilidad también varía desde un acuerdo pobre a un acuerdo muy bueno (ICC $=.27-.56)$.

Como puede verse, si bien el uso de la CTS es frecuente, se necesitan más investigaciones que indaguen la confiabilidad de la escala. A nivel internacional los estudios sobre este tópico son escasos y en Argentina no se evidencian investigaciones previas. Contar con un instrumento fiable para medir la competencia de los terapeutas en el contexto de la terapia cognitivo-conductual resulta una herramienta de gran valor a nivel clínico y empírico (Muse \& McManus, 2013), motivo por el cual el presente trabajo tiene como principal objetivo indagar la confiabilidad de la escala CTS. 


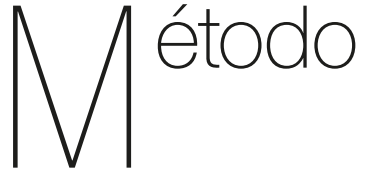

\section{Participantes}

La muestra quedó conformada por 10 jueces expertos. Se trabajó con terapeutas de ambos sexos (70\% mujeres; 30\% hombres), con edades comprendidas entre los 28 y 43 años (media de edad = 34.5 años). Como criterio de selección se incluyó: a) que tuvieran más de 5 años de experiencia, y b) formación acreditada en TCC. Dichos jueces evaluaron el desempeño que presentaban terapeutas cognitivo-conductuales ( $n=4$ ) durante el desarrollo de una sesión. La selección de los profesionales se llevó a cabo mediante un muestreo no probabilístico intencional.

\section{Instrumentos}

\section{Escala de Terapia Cognitiva}

(Cognitive Therapy Scale, CTS). Está conformada por 11 ítems con un formato tipo rating a partir de lo cual un observador puntúa las competencias evidenciadas por un terapeuta en una sesión. El puntaje varía en una escala de 0 (pobre desempeño) a 6 (excelente desempeño). Los primeros seis ítems permiten clasificar las Competencias Terapéuticas Generales (i.e. establecimiento de agenda; feedback; entendimiento; efectividad interpersonal; colaboración; y uso del tiempo). El restante grupo de 5 ítems permite evaluar las Habilidades Terapéuticas
Cognitivas específicas (i.e. descubrimiento guiado; foco en las cogniciones o comportamientos claves; estrategia de cambio; aplicación de técnicas cognitivoconductuales; y plan de acción). La escala cuenta con evidencia de validez y confiabilidad (Vallis et al., 1986) así como consistencia interjueces (Barber et al., 2003; Gordon, 2006). De manera general puede indicarse que la CTS demuestra una consistencia interna óptima (valores $\alpha$ entre .84 y .95; y correlaciones ítem-total entre .48 y .94), así como una estructura bifactorial (Muse \& McManus, 2013; Vallis et al., 1986).

\section{Procedimiento}

El presente estudio sigue un diseño de tipo instrumental (Montero \& León, 2007). Para desarrollar este trabajo se contrató a un actor profesional para que representara un paciente con sintomatología depresiva. La decisión de trabajar con un actor y no con un paciente real obedeció fundamentalmente a motivos éticos. En efecto, ninguno de pacientes consultados accedió a filmar una sesión terapéutica con propósitos de investigación. Al no contar con el consentimiento informado de los pacientes se consideró oportuno entrenar a un actor para que simulara sintomatología depresiva. Concretamente se solicitó que simulara síntomas cognitivos (visión negativa de sí mismo, los demás y el futuro), conductual (llanto, verbalizaciones de autocrítica, conductas que denotan fatiga) y afectivos (tristeza, perdida de energía e interés) propios de un trastorno depresivo mayor.

Cada terapeuta tuvo una sesión de una hora con el mismo actor. Dichas 
sesiones terapéuticas fueron filmadas y enviadas a 10 jueces expertos, quienes evaluaron de forma independiente cada uno de los videos. Es decir, que cada juez (10 en total) analizó un total de cuatro videos (uno por terapeuta), efectuándose un total de 40 mediciones.

Los datos fueron cargados en el programa SPSS 21, y se realizaron los análisis de confiabilidad. La confiabilidad de la escala fue verificada mediante tres pruebas, a través de la correlación ítem-total corregida, el coeficiente alfa de Cronbach, y la correlación inter-clase utilizando un modelo mixto (efectos aleatorios de las personas y efectos fijos de los ítems).

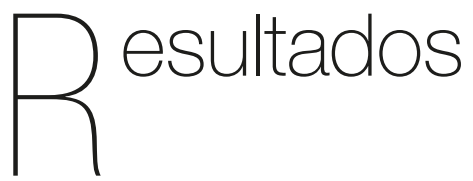

En primer lugar, se detalla la consistencia interna observada para el conjunto de ítems. En la tabla 1 queda especificada la correlación ítem total corregida, así como el coeficiente alfa de Cronbach obtenido.

Tabla 1.

Correlación Ítem-Total Corregida y Alfa de Cronbach: Escala Total y Subescalas

\begin{tabular}{|c|c|c|c|}
\hline Subescala e Ítems & CTS Total & Habilidades Generales & Habilidades TC \\
\hline \multicolumn{4}{|l|}{ Habilidades Generales } \\
\hline Agenda & .807 & .749 & \\
\hline Feedback & .912 & .851 & \\
\hline Comprensión & .983 & .987 & \\
\hline Efectividad Interpersonal & .732 & .731 & \\
\hline Cooperación & .976 & .983 & \\
\hline Tiempo & .978 & .960 & \\
\hline \multicolumn{4}{|l|}{ Habilidades TC } \\
\hline Descubrimiento Guiado & .943 & & .889 \\
\hline Focousing & .991 & & .998 \\
\hline Estrategias para el Cambio & .979 & & .989 \\
\hline Aplicación de Técnica & .984 & & .993 \\
\hline Tareas & .971 & & .980 \\
\hline Alfa de Cronbach & .974 & .934 & .956 \\
\hline
\end{tabular}

Nota. Habilidades TC $=$ Habilidades de la Terapia Cognitiva.

Tal como puede observarse, en la Tabla 1 se presentan correlaciones altas en todos los ítems, obteniéndose una consistencia interna óptima [12], tanto para la escala total como para las subescalas correspondientes.
Finalmente se muestra la consistencia inter-jueces calculada mediante la correlación inter-clase (ICC; Tabla 2). 
Tabla 2.

Correlación Interclase según Competencias Específicas y Puntaje Total.

\begin{tabular}{|c|c|}
\hline Subescala e Ítems & ICC \\
\hline Habilidades Generales & .818 \\
\hline Agenda & .643 \\
\hline Feedback & .653 \\
\hline Comprensión & .692 \\
\hline Efectividad Interpersonal & .830 \\
\hline Cooperación & .744 \\
\hline Tiempo & .651 \\
\hline Habilidades TC & .831 \\
\hline Descubrimiento Guiado & .628 \\
\hline Focousing & .605 \\
\hline Estrategias para el Cambio & .660 \\
\hline Aplicación de Técnica & .809 \\
\hline Tareas & .740 \\
\hline Puntaje Total & .842 \\
\hline
\end{tabular}

La Tabla 2 da cuenta de valores de ICC entre moderados y muy buenos cuando se consideran los ítems, y valores ICC entre buenos y muy buenos para los puntajes totales.

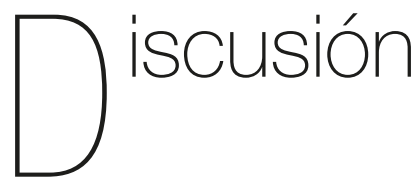

Contar con instrumentos fiables para medir la competencia de los terapeutas en el contexto de la TCC resulta de una herramienta de gran valor a nivel clínico y empírico (Muse \& McManus, 2013). A nivel clínico posibilita verificar si los psicoterapeutas son competentes para llevar a cabo sesiones clínicas óptimas. A nivel empírico permite indagar si estas competencias pueden mejorarse luego de ciertas intervenciones, o si las mismas se relacionan con mejoras en la sintomatología de los pacientes. La CTS es una de las escalas más ampliamente utilizadas con el objeto de medir la competencia terapéutica (Barber et al., 2003; Gordon, 2006; Muse \& McManus, 2013). A nivel internacional no son muchos los antecedentes que se han propuesto indagar su validez y confiabilidad, y la confiabilidad de la escala sigue siendo un tema controvertido (Muse \& McManus, 2013; Reichelt et al., 2003). Sumado a lo anterior, no se encuentran investigaciones de este tipo en Argentina, motivo por el cual el presente trabajo tuvo como principal objetivo indagar la confiabilidad de la escala CTS.

Los resultados permiten concluir que se trata de un instrumento confiable. En efecto se aprecia una consistencia interna óptima, reflejado esto tanto en la correlación ítem-total como en el coeficiente alfa de Cronbach. Asimismo, se observó en general una buena consistencia en las puntuaciones dadas por los diferentes profesionales. Estos datos son consistentes con aquellas investigaciones que han dado cuenta de un acuerdo moderado, bueno y muy bueno, al medir la confiabilidad interjueces del CTS (Muse \& McManus, 2013; Reichelt et al., 2003). Según Muse \& MacManus (2013) la razón de que no se encuentre consistencia entre los resultados de los diversos estudios puede deberse a algunos de los siguientes factores externos al instrumento: diferencia en el número de evaluadores, y diferencias en su experiencia y entrenamiento. Más allá de estas consideraciones, los datos presentados en esta investigación avalan la utilización de la escala en el contexto argentino, y permiten señalar que la CTS es una escala confiable. 
Para concluir es posible afirmar que se cuenta con una herramienta de evaluación útil para la formación y el entrenamiento en habilidades terapéuticas. Asimismo, a partir de este instrumento se posibilita la puesta en marcha de nuevas líneas de investigación en Argentina, que permitan evaluar el papel de las habilidades comunicacionales del terapeuta en la eficacia terapéutica.

Futuras investigaciones podrían indagar la validez convergente de la escala, para analizar si la percepción de competencias observadas por jueces expertos, también se conduce con la autopercepción que mantienen los terapeutas sobre sus competencias. $Y$ en segundo lugar, trabajar acerca de la validez externa del instrumento, realizando la aplicación del instrumento en contextos clínicos reales y manteniendo constantes ciertas variables (número de sesión evaluada, cantidad de tiempo registrado, problemática de los pacientes, etc.) (Fernández-Álvarez et al., 2015).

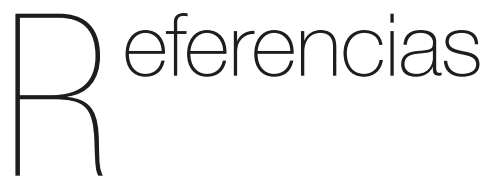

American Psychological Association. (2005). Policy Statement on Evidence-Based Practice in Psychology. Recuperado de http://www.apa.org/practice/guidelines/ evidence-based-statement.aspx

Araya, C. \& Herrera, P. (2007). Errores y dificultades comunes de terapeutas novatos. (Tesis de Magister inédita). Universidad Adolfo Ibáñez, Santiago, Chile.
Barber, J. P., Liese, B. S., \& Abrams, M. J. (2003). Development of the Cognitive Therapy Adherence and Competence Scale. Psychotherapy Research, 13(2), 205-221. http://dx.doi.org/10.1093/ptr/ kpg019

Barnfield, T. V., Mathieson, F. M., \& Beaumont, G. R. (2007). Assessing the development of competence during postgraduate cognitive-behavioral therapy training. Journal of Cognitive Psychotherapy, 21(2), 140-147. https://doi. org/10.1891/088983907780851586

Beck, A. T., Rush, A. J., Shaw, B. F., \& Emery, G. (1976). Terapia cognitiva de la depresión. Bilbao: Desclée de Brower.

Bennett-Levy, J. (2006). Therapist skills: A cognitive model of their acquisition and refinement. Behavioural and Cognitive Psychotherapy, 34(01), 57-78. https://doi. org/10.1017/S1352465805002420

Casari, L., Ison, M.S., Albanesi, S. \& Maristany, M. (2017). Panorama actual de investigación sobre el terapeuta en psicoterapia. Acta Psiquiátrica y Psicológica de América Latina, 63(2), 104-114.

DeRubeis, R. J., Hollon, S. D., Evans, M. D., \& Bemis, K. M. (1982). Can psychotherapies for depression be discriminated? A systematic investigation of cognitive therapy and interpersonal therapy. Journal of Consulting and Clinical Psychology, 50(5), 744-756. http://dx.doi. org/10.1037/0022-006X.50.5.744

Fairburn, C. G., \& Cooper, Z. (2011). Therapist competence, therapy quality, and therapist training. Behaviour Research and Therapy, 49(6), 373-378. https://doi.org/10.1016/j. brat.2011.03.005 
Fernández-Álvarez, H., García, F., Lo Bianco, J., \& Corbella, S. C. (2003). Assessment questionnaire on the personal style of the therapist PST-Q. Clinical Psychology \& Psychotherapy, 10, 116-125. doi: 10.1002/ cpp.358.

Fernández-Álvarez, H., Castañeiras, C., Curtarelli, A., García, F., Gómez, B., Lichtenberger, A., \& Corbella, S. (2015). Presentación de una Guía para la Observación y Clasificación de la Conducta Verbal de los Terapeutas. Terapia Psicológica, 33(1), 23-34. http://dx.doi. org/10.4067/S0718-48082015000100003

Gordon, K. P. (2006). A comparison of two versions of the cognitive therapy scale. Behavioural and Cognitive Psychotherapy, 35, 343-53. doi: 10.1017/ S1352465806003390

Heinonen, E. (2014). Therapists' professional and personal characteristics as predictors of working alliance and outcome in psychotherapy. Accademic Dissertation. Research/National Institute for Health and Welfare. Recuperado de http://thl32-kk.lib. helsinki.fi/bitstream/handle/10024/114948/ URN_ISBN_978-952-302-127-3. pdf?sequence=1

Hill, C. E., O'Grady, K. E., \& Elkin, I. (1992). Applying the Collaborative Study Psychotherapy Rating Scale to rate therapist adherence in cognitive-behavior therapy, interpersonal therapy, and clinical management. Journal of Consulting and Clinical Psychology, 60, 73-79.

Holdsworth, E., Bowen, E., Brown, S., \& Howat, D. (2014). Client engagement in psychotherapeutic treatment and associations with client characteristics, therapist characteristics, and treatment factors. Clinical Psychology Review, 34(5), 428-450.

James, I. A., Blackburn, I. M., Milne, D. L., \& Reichfelt, F. K. (2001). Moderators of trainee therapists' competence in cognitive therapy. British Journal of Clinical Psychology, 40(2), 131-141. DOI: 10.1348/014466501163580

Mairal, J. B. (2009). Habilidades Clínicas en la Terapia Conductual de Tercera Generación. Clínica y Salud, 20(2), 109-117.

Muse, K., \& McManus, F. (2013). A systematic review of methods for assessing competence in cognitive-behavioural therapy. Clinical Psychology Review, 33(3), 484-499. https://doi.org/10.1016/j. cpr.2013.01.010

Myles, P. J., \& Milne, D. L. (2004). Outcome evaluation of a brief shared learning programme in cognitive behavioural therapy. Behavioural and Cognitive Psychotherapy, 32(2), 177-188. https://doi. org/10.1017/S1352465804001183

Nissen-Lie, H. A., Havik, O. E., Høglend, P. A., Monsen, J. T., \& Rønnestad, M. H. (2013). The contribution of the quality of therapists' personal lives to the development of the working alliance. Journal of Counseling Psychology, 60(4), 483-495. doi: 10.1037/ a0033643.

Rakovshik, S. G., \& McManus, F. (2010). Establishing evidence-basedtraining in cognitive behavioral therapy: A review of current empirical findings and theoretical guidance. Clinical Psychology Review, 30(5), 496-516. http://dx.doi.org/10.1016/j. cpr.2010.03.004

Montero, I. \& León, O. G. (2007). A guide for naming research studies in Psychology. 
International Journal of Clinical and Health Psychology, 7(3), 847-862.

Reichelt, F. K., James, I. A. \& Blackburn, I.M. (2003). Impact of training on rating competence in cognitive therapy. Journal of Behavior Therapy and Experimental Psychiatry, 34, 87-99. doi: 10.1016/ S0005-7916(03)00022-3

Rodríguez, M. L. \& Salinas, J. L. (2011). Entrenamiento en habilidades terapéuticas: algunas consideraciones. Enseñanza e Investigación en Psicología, 16(2), 211-225.

Romero, A. F. (2008). Factores atribucionales de la efectividad psicoterapéutica. Anales de Psicología, 24(1), 88-99. Recuperado de http://www.redalyc.org/articulo. oa?id=16724111

Roth, A.D. \& Pilling, S. (2007). The competences required to deliver effective cognitive and behavioural therapy for people with depression and with anxiety disorders. Department of Health: London.

Shaw, B. F., \& Wilson-Smith, D. (1988). Training therapists in cognitive-behaviour therapy. In C. Perris, I. M. Blackburn, \& H. Perris (Eds.), Cognitive psychotherapy: Theory and practice. Heidelberg: Springer Verlag.

Shaw, B. F., Elkin, I., Yamaguchi, J., Olmsted, M., Vallis, T. M., Dobson, K. S, \& Imber, S. D. (1999). Therapist competence ratings in relation to clinical outcome in cognitive therapy of depression. Journal of Consulting and Clinical Psychology, 67(6), 837-846. http://dx.doi. org/10.1037/0022-006X.67.6.837

Strunk, D., Brottman, M., De Rubeis, R., Hollon, S. (2010). Therapist Competence in Cognitive Therapy for Depression:
Predicting Subsequent Symptom Change. Journal of Consulting and Clinical Psychology, 78(3), 429-437. http://dx.doi. org/10.1037/a0019631

Vallis, T. M., Shaw, B. F., \& Dobson, K. S. (1986). The Cognitive Therapy Scale: Psychometric properties. Journal of Consulting and Clinical Psychology, 54(3), 381-385.http:// dx.doi.org/10.1037/0022-006X.54.3.381

Webb, C. A., DeRubeis, R. J., \& Barber, J. P. (2010). Therapist adherence/competence and treatment outcome: A meta-analytic review. Journal of Consulting and Clinical Psychology, 78(2), 200 - 2011. http://dx.doi. org/10.1037/a0018912

Whisman, M. A. (1993). Mediators and moderators of change in cognitive therapy of depression. Psychological Bulletin, 114(2), 248-265. http://dx.doi. org/10.1037/0033-2909.114.2.248

Winkler, M. I., Cáceres, C., Fernández, I., \& Sanhueza, J. (1989). Factores inespecíficos de la psitoterapia y efectividad del proceso terapéutico: una sistematización. Revista Terapia Psicológica, 8(11), 34-40.

Yáñez-Galecio, J. (2005). Competencias Profesionales del Psicólogo Clínico: Un Análisis Preliminar. Terapia Psicológica, 23(2), 85-93. Recuperado de http://www. redalyc.org/articulo.oa?id=78523209

Young, J. \& Beck, A. T. (1980). Cognitive Therapy Scale rating manual (Unpublished manuscript). University of Pennsylvania, Philadelphia. 